\title{
TWO BEAM PROTON ACCELERATOR FOR NEUTRON GENERATORS AND ELECTRONUCLEAR INDUSTRY
}

\author{
G.V. Dolbilov, JINR, Dubna, 141980, Russia
}

\section{Abstract}

A concept of a linear proton accelerator with $\mathrm{RF}$ cavities excited by the electron beam is discussed. The accelerators with parameters of the second proton beam equal to $1 \mathrm{GeV}, 10-100 \mathrm{~mA}$, can be used for a new type of electronuclear installations and neutron productions.

\section{INTRODUCTION}

Actually all RF accelerators are two beam accelerators. In these accelerators the kinetic energy of electron beams is converted into the energy of high frequency oscillations in RF sources to drive accelerating modules and increase the energy of accelerated particles. In conventional RF accelerators the RF power passes through a feeding waveguide, wave mode transformers and vacuum windows of the RF source and the accelerating structure which have a relatively high wave impedance. Increasing the RF power can result in the over-voltage in these devices of the power transmission. The future accelerators will require such a level of the RF power which is limited by this over-voltage. The e-beam can be used to excite the accelerating structure directly without the excitation of the electrodynamic structure of the RF source. In this case the units of the RF power transmission are absent and the maximum $\mathrm{RF}$ voltage is conditioned by the loaded impedance of the accelerating structure and the maximum accelerating gradient depends only on its electric strength. The value of this gradient may by very high. For example, at the frequency of $30 \mathrm{GHz}$ the single-cell cavity excited by e-beam was operating without breakdown at the peak accelerating gradient of $290 \mathrm{MV} / \mathrm{m}$. The cavity surface electric field was more than of $750 \mathrm{MV} / \mathrm{m}$ [1].

\section{ONE-CHANNEL ACCELERATOR}

In this two beam accelerator the driving e-beam and the main p-beam are passing through one and the same channel of the electrodynamic structure which bunches the driving e-beam and simultaneously accelerates the main p-beam. Since the one mode regime of the accelerator-buncher structure can be realized, all parasitic modes of self-excitation might be suppressed [2], including modes of the beam-beam instability. If energy losses of the driving e-beam are covered by the external electric field, the efficiency of the conversion of the electron kinetic energy into the proton energy can be close to 1. A scheme of the one-channel two-beam induction accelerator is shown in Fig. 1.
For effective energy conversion, the solution of 3 principal tasks is required:

- longitudinal stability of the driving electron bunches;

- high amplitude of the RF field excited by the electron beam;

- $\quad$ synchronism of the accelerated protons and excited RF fields.

\subsection{Longitudinal Stability of the Electron Bunches}

Bunching the electrons is provided by the excited RF fields similar to the multi-cavity klystron buncher. The buncher frequency is slightly less than the frequency of the unloaded cavities of the accelerating structure and the cavities have almost a pure inductive impedance, so the bunches of the driving electron beam are passing through the cavities at an almost zero phase of the RF field. As a result of the phase shift between the excited voltage and the electron bunches, the longitudinal stability is provided.

To verify a possibility of the stationary bunches, a computer simulation has been performed. The results of the simulation are shown in Fig. 2.

The computer simulation has shown that there are optimum matching regimes, when the longitudinal dimension of the bunches is matched with the electrodynamic structure of the accelerator and the oscillation amplitude of the electron bunches has a minimal value. In one of these matching regimes the 500 $\mathrm{A}, 1000 \mathrm{keV}$, the electron beam excites the $\sim 1 \mathrm{MV}$ voltage in the $14 \mathrm{GHz}$ cavities (Fig. 2). Herewith the external induction field, which covers the electron energy loss, is equal to $10 \mathrm{kV}$ per cavity (or $\sim 1 / 100$ part of the excited RF voltage). The average proton accelerating gradient is determined by the number of the cavities per unit of length.

\subsection{Condition of Synchronism for Protons and Excited Fields}

Synchronism for the proton beam and the excited RF fields is provided by appropriate spacing of the accelerator-buncher RF structure. The RF structure period should be equal to: [3-5]

$$
L=k \lambda /\left(\beta_{p}^{-1} \mp \beta_{e}^{-1}\right),
$$

where $k$ is integer, $\lambda$-wave length, $\beta_{p, e}$ - relative velocity of the protons and electrons. 


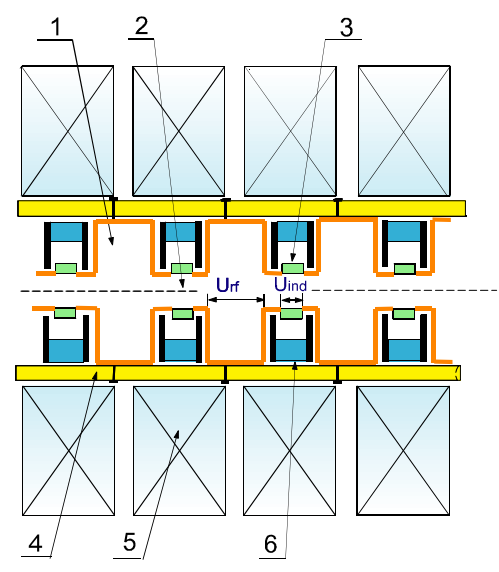

Figure 1: One of possible schemes of the electrodynamic structure of a Two-Beam Induction Linac. Here: 1-rf cavity, 2-drift tube, 3-rf absorbing insertion, 4-ceramic tube, 5-induction core, 6-permanent magnet of the focusing system

\subsection{Cavity Excitation}

Using the law of the energy conservation

$$
P_{e}=P_{p}+P_{\text {loss }},
$$

we can find that the amplitude of the excited RF voltage in the cavity is equal to $[3,5]$

$$
U_{e}=\left(1-\eta_{e}\right) 2 \rho Q_{0} I_{e} M_{e} / \sqrt{1+\xi^{2}},
$$

where $P_{e}$ - average power of the driving e-beam which is sent to excite the cavity, $P_{p}$ - average power which is taken to accelerate the proton beam, $P_{\text {loss }}$ - power loss in the cavity, $I_{e}$ - average beam current, $Q_{0}$ - unloaded quality factor, $\eta_{e}=1-Q_{\text {load }} / Q_{0}$ - efficiency of the energy conversion from the driving e-beam into the $\mathrm{p}$ beam.

Relative detuning of the cavity is $\xi=Q_{\text {load }}(2 \Delta f / f)$. The interaction factor of the electron and the cavity is $M_{e}=\left[\sin \left(\theta_{e} / 2\right)\right] /\left(\theta_{e} / 2\right), \theta_{e}$ is an electron-transit angle and $\rho$ is the wave impedance of the cavity. The phase shift of the induced RF voltage is $\varphi_{e}=-\operatorname{arctg} \xi$.

An equivalent deaccelerating voltage for the e-beam which should be recovered by the external induction field is:

$$
U_{\text {ind }}=U_{e} \cos \varphi_{e}=U_{e} / \sqrt{1+\xi^{2}}
$$

Thus, the gain of an accelerating gradient for the protons in the two beam accelerator is equal to

$$
E_{e} / E_{\text {ind }}=\sqrt{1+\xi^{2}} \cong \xi .
$$

\section{TWO (OR MULTI) CHANNEL ACCELERATOR}

Cross sections of the two and multi channel two-beam accelerator are shown in Fig. 3.

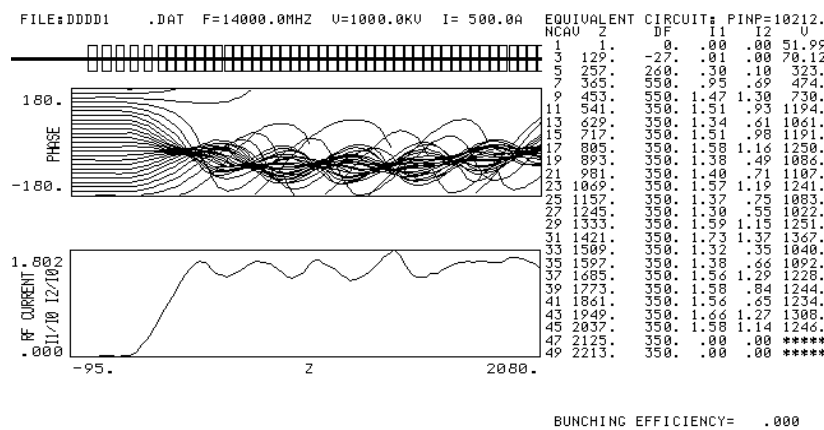

Figure 2: Results of computer simulations of the phase trajectories of electron in the electrodynamic structure of the two-beam accelerator, first harmonic of the current of the bunched e-beam and rf voltage excited by e-beam in cavities (right column $-U_{(k V)}$ ).

The driving electron beam excites the e-cavities and is bunched by their RF fields.

The protons are accelerated by RF fields of the $p$ cavities, which have magnetic coupling with e-cavities.

A resonance frequency of the p-cavities is equal to the bunching frequency. To keep the longitudinal dimension of the electron bunches, the relative detuning of the ecavities is equal to $\xi>>1$.

The two (multi) channel accelerator design is more complicated, but it has a higher gain of the accelerating gradient.

\subsection{Excited Voltage}

The voltage of the p-cavity is: $U_{p}=\chi U_{e}$

The voltage transmission gain is:

$$
\chi=\left(1-\eta_{p}\right) k_{m} \sqrt{\rho_{p} / \rho_{e}} Q_{0, p},
$$

where $\rho_{p, e^{-}}$wave impedance of the p,e-cavities, $k_{m}$ magnetic coupling coefficient of the e- and p-cavities, $\eta_{e, p}=1-\left(Q_{\text {load }} / Q_{0}\right)_{e, p}$ is the efficiency of the ebeam/RF and RF/p-beam energy conversion in the cavities, $Q_{\text {load }}$ and $Q_{0}$ - loaded and unloaded quality factor.

The gain of the proton accelerating gradient is:

$$
E_{p} / E_{\text {ind }}=\chi \sqrt{1+\xi^{2}} .
$$

\section{AVERAGE ACCELERATING GRADIENT}

The average proton accelerating gradient $E_{a, p}=U_{p} / L$ is:

$$
E_{a, p}=E_{p, \max }(h / L) M_{p},
$$

where $h$ - accelerating gap, $L$ - RF structure period.

Analytical calculations, computer simulations and the high gradient test [1] have shown very high accelerating gradients of the two-beam accelerator. As a matter of fact, 
the maximum accelerating gradient is limited by the electrical strength ( $E_{p, \max }$ ) of the RF structure.

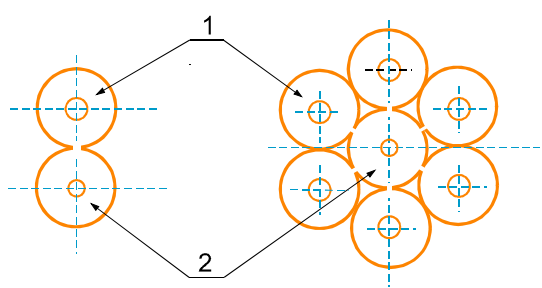

a

b

Figure 3: Cross section of the two-channel (a) and multi-channel (b) two-beam accelerator. Here 1 - e-beam cavities, 2 -p-beam cavities.

The main maximum of the averaging parameter $\alpha=M_{p} h / L$ is equal to $\sim 0.6$ at the proton-transit angle $\theta_{p}=\pi$. Other maxima reduce slowly at higher transit angles.

As for conventional accelerators, the frequencies of the initial and final parts of the two-beam proton accelerator can be different to decrease the transit angle at a low proton energy. However, there is a possibility to use one and the same frequency in all parts of the two-beam accelerator. For example, at $E_{p, \max } \approx 100 \mathrm{MeV} / \mathrm{m}$ and an operating frequency $f=11.4 G H$, the average accelerating gradient will be equal approximately to:

$60 \mathrm{MeV} / \mathrm{m}$ - for the final accelerator part,

$3-6 \mathrm{MeV} / \mathrm{m}$ - for the initial accelerator part

The two-beam method of the proton acceleration allows one to use a standing-wave type of the accelerating structure in the final (main) part of the accelerator. A scheme of this structure is shown in Fig. 4.

The average proton and electron accelerating gradient, the average current of the e- and p-beam and the conversion efficiency of their energy are associated by the following ratio:

$$
E_{a, p} / E_{a, e}=\eta M_{e} I_{e} / M_{p} I_{p}=\chi \sqrt{1+\xi^{2}} .
$$

\section{INTENSITY OF ELECTRON AND PROTON BEAMS}

To reduce the relative value of the energy loss in the cavity and increase the efficiency of the beam-beam energy conversion, the average current of the proton beam should be increased up to

$$
I_{p}=\eta \frac{I_{e}}{\chi \sqrt{1+\xi^{2}}} \frac{M_{e}}{n M_{p}},
$$

where $n$ - a number of the proton bunches accelerated together in one cavity, when $\theta_{p}=(3,5 \ldots) \pi$.

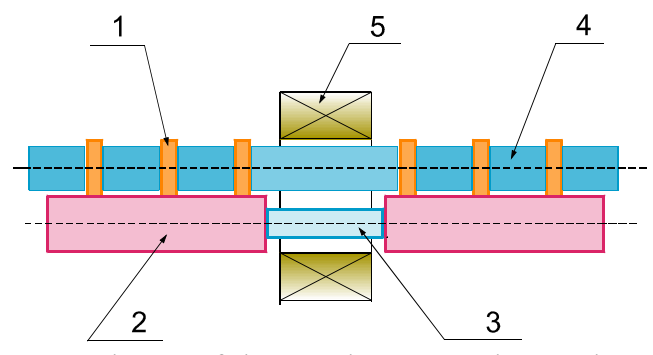

Figure 4: Scheme of the two-beam accelerator based on a standing-wave type or multi-cell resonator proton accelerating structure. Here: 1 - e-beam cavities; 2 - pbeam accelerating structure; 3,4 - focusing system permanent magnet of $\mathrm{p}$ - and e-beam channels; 5 - core of induction module.

At this current of the proton beam the efficiency of this energy conversion will be equal to the chosen value of $\eta=\eta_{e} \eta_{p}$.

For the $100 \mathrm{~mA}, 1 \mathrm{GeV}$ proton accelerator and accelerating gradient gain $E_{a, p} / E_{\text {ind }}=10^{2}-10^{3}$, the average current of the e-beam is required to be $\sim 10-100 A$.

The induction module is a pulsed device, so the twobeam accelerator should operate in the pulsed regime. It allows one to reach the higher level of the beam-beam energy conversion [5], that is important for the low current and "room temperature" accelerators. The average e- and p-beam currents depend on the repetition rate of the two-beam accelerator.

\section{ACKNOWLEDGEMENTS}

The author is grateful to V.E. Teryaev for upgrading the klystron computer program to adapt one for the twobeam accelerator simulation.

\section{REFERENCES}

[1] W. Wuensch et al., "A Very-High-Gradient Test of $30 \mathrm{GHz}$ Single-Cell Cavity," Proc. of EPAC 2000, Vienna, Austria, pp. 271-273

[2] G.V. Dolbilov, V.E. Balakin et al., "A concept of a Wide Aperture Klystron with RF Absorbing Drift Tubes for a Linear Collider," Nuclear Instruments and Methods in Physics Research, A,383, pp. 318324 (1996)

[3] G.V. Dolbilov, "Two-Beam Induction Linear Collider," Proc. of EPAC 2000, Vienna, Austria, p. 904

[4] G.V. Dolbilov, "High Current Linacs at JINR and Perspective of Their Application for acceleration of Ions," AIP Conference proceedings 480, "Space Charge Beam Physics for Heavy Ion Fusion," Saita, Japan, 1998, pp.85-89

[5] G.V. Dolbilov, "Two-Beam Accelerator for Electronuclear Industry," In "Problems of Atomic Science and Technology, part 2, Plasma Electronic and New Methds of Acceleration," 2000, N2 (36), pp. $108-110$ 